\title{
A Lingua Franca for Security by Design
}

\author{
Alexander van den Berghe*, Koen Yskout*, Riccardo Scandariato ${ }^{\dagger}$ and Wouter Joosen* \\ *imec-DistriNet, KU Leuven, 3001 Leuven, Belgium \\ \{alexander.vandenberghe,koen.yskout,wouter.joosen\}@cs.kuleuven.be \\ ${ }^{\dagger}$ Software Engineering Division, Chalmers and Göteborg University, 41756 Göteborg, Sweden \\ riccardo.scandariato@cse.gu.se
}

\begin{abstract}
The principle of security by design is advocated by academia as well as industry. Unfortunately, its adoption in practice is not yet widespread. We believe a reason for this is the lack of a 'lingua franca' for security modelling. Such a language should support security specialists to precisely describe the security aspects in a software design, as well as simultaneously serve to communicate with a broader audience of stakeholders. For this paper, we have assessed how well a formally backed security modelling language we previously proposed, suits the needs of the needs of these two groups. Concretely, we report on a large user study investigating how well security novices are able to comprehend the foundations of our language. Furthermore, to assess our language's practicality, we show how it can be used to create a realistic model of authentication. We have found that our language's foundations are comprehensible to a broader audience and they allow to precisely model a design's security aspects, albeit some shortcomings requiring attention have been identified. Based on these findings, we believe that a precise yet comprehensible security by design lingua franca is within reach.
\end{abstract}

Index Terms-Security by design, Modelling language, User study, Evaluation

\section{INTRODUCTION}

Comprehensively managing software security requires tackling it from the early phases in the development life cycle, as advocated by the security by design principle. An exemplary illustration of the viciousness of security design flaws is provided by Microsoft's Active Directory vulnerability [1]. Fixing this design flaw, rated as critical, required comprehensive architectural changes that took a year to be developed. Tackling security early on requires the ability to create and reason about a high-level security view of the software. As a prerequisite for this, software designers should have access to a suitable modelling language that facilitates expressing and reasoning about the security aspects of their design.

Such a security modelling lingua franca has to support the objectives of several stakeholders. A company, especially a medium to large one, typically has a specialised security team responsible to assess and harden the security of the designed software. These security experts require a very precise security view of the design to facilitate in-depth reasoning, yet they also use this security view to communicate with, for example, software architects, managers, and quality attribute leaders.

As such, security views are created by a small, specialised team but need to be read and understood by a much larger audience across several departments. With the backup of our experience at large model-driven companies, we argue that, from a company perspective, educating this larger audience to understand the semantics of such a view is more costly and time-consuming than, for instance, hiring a $\mathrm{PhD}$ as the security specialist who creates the models.

The security modelling languages proposed by research mostly focus on providing the required level of precision for security specialists, often by relying on formal methods [2], [3], [4]. Such languages facilitate to meticulously analyse whether security requirements are upheld by formally expressing both requirements and designed solutions. Unfortunately, as often pointed out, "formal methods involve significant human labour, and are expensive" [5]. A recurring concern is thus that introducing formally founded languages cannot be applicable to the industrial reality due to their complexity and required skill level. For similar reasons, it has also been hard to catch the interest of industrial partners for such techniques.

In order for any language to be broadly adopted as security modelling lingua franca, it has to reconcile two contradicting forces: (1) it should possess sufficiently precise semantics to allow security experts to design and analyse security solutions; and (2) the designed solutions should be comprehensible to a broader audience of security novices.

In this paper, we assess to what extend a security modeling language we previously proposed [4] (briefly explained in Section III), succeeds in balancing these two opposing forces. More specifically, we have conducted a descriptive study (Section IV) in which 105 participants, software engineering students with limited security expertise, completed several tasks using the language, in order to asses its comprehensibility to a broader audience. In addition, we have applied our language to realistically model the authentication mechanism commonly used on the web, comprising usernames and passwords, sessions, two-factor authentication, registration, the possibility for users to change their password, and account recovery. In this paper we use excerpts of this elaborate design to illustrate how the language's building blocks can be used by security experts to create precise security views (Section V).

Based on our findings, we believe that a precise and still broadly comprehensible lingua franca for security by design, based on the evaluated language, is within reach. The main contributions of this paper consist of (1) an in-depth analysis whether a particular security modelling language can reconcile the required precision with comprehensibility; and (2) a design for a realistic authentication mechanism, which can possibly be used as case study for comparing other language proposals. 
We hope that our results inspire the security engineering community to similarly assess and compare other candidate languages with respect to the different audiences such a language must target.

\section{RELATED WORK}

Several systematic literature reviews [6], [7] show that various security modelling languages have been proposed over the years. Here we limit ourselves to the most important ones.

UMLsec [2] extends UML with a security profile and a formal semantics, which allows formal tools, e.g. model checkers, to verify security properties. Similarly, SecureMDD [3] provides a UML profile for security used to generate formal models, based on which security properties can be proven using an interactive theorem prover. Although both approaches have been applied to large systems, even in industrial case studies, this happened almost exclusively in close collaboration with the original authors. Whether these languages can serve to communicate with a broader audience remains to be evaluated.

For some other security-oriented languages, user studies have been done. Heyman et al. [8] leverage Alloy to formally model security solutions, although it requires designers to specify all security aspects themselves. Based on a small user study, in which two participants extended an architecture using five formally modelled security patterns, the authors conclude that their technique is useful and increases the designers' confidence in the security of the system.

SecDSVL [9] supports modelling and assessing security mechanism at an enterprise level, focusing on security management. It lacks the precision to precisely design security solutions during the software design phases. The authors conclude that their language is usable based on the results of a user study in which seven participants rated it along several dimensions, such as ease of use and complexity after using it.

Giordano et al. [10] propose a language to model rolebased access control policies, which is thereby narrower in scope than our language. In the author's usability study, 60 participants (volunteers with mixed backgrounds) were given a short introduction to the language and accompanying tool, after which they were asked to model a freely chosen access control policy. The authors conclude that their language is usable based on the answers to a questionnaire.

In practice, a popular notation for security analysis is a Data Flow Diagram (DFD) [11], used in the STRIDE [12, chapter 3] threat modelling approach. Despite their popularity, DFDs lack a precise semantics and are not security-oriented, requiring designers to add (security) semantics themselves.

Primarily situated on the formal side, approaches such as CSP [13], [14], the applied Pi calculus [15], CryptoVerif [16], and ProVerif [17] do provide the required degree of precision, and have also been successfully used to prove security properties. We are, however, not aware of any comprehensibility studies for these approaches with a novice audience (and are a bit pessimistic about the eventual results thereof). Finally, Spi2JavaGUI [18] does combine a graphical modelling language with formal analysis but is limited to security protocols.

\section{BACKGRound on Secure Modelling LANGUAGE}

In this section, we briefly introduce our modelling language; a detailed, complete overview, together with a formal specification, can be found in [4]. Our language essentially consists of four primary concepts: data that is manipulated by processes, which in turn can collaborate by grouping them into networks, each of which can be further refined by assumptions.

\section{A. Data}

Data elements can be represented by one of several (mostly security-specific) data types, including generic plain data, encrypted data, identities credentials, and cryptographic keys. For instance, symk 3 represents a symmetric cryptographic key, where 3 functions as an identifier. enc (id 5) (symk 4) represents the encryption of an identity id 5 using the symmetric key symk 4. More complex data structures can be constructed by (recursively) gathering data elements in collections. Data elements are considered equal if they are of the same type and have identical identifiers, or (for more complex types) if their contained data elements are (recursively) equal.

\section{B. Processes}

The bulk of our language's semantics is encapsulated in 23 pre-defined processes, making these its heart. Each process exhibits a single, well defined function, e.g. encrypting data. The internal state of a process is formalised as a, possibly nondeterministic, state machine combined with input and output queues through which data can respectively enter and leave the process in a first-in-first-out fashion.

For example, an Encrypter process reads data from a data input queue, encrypts it using a cryptographic key and writes the result to an output queue. When a cryptographic key is provided via its designated key input queue, the Encrypter can read the key and configure it as its current encryption key, replacing any previously used key. If a process has inputs in multiple queues, it can non-deterministically choose in which order to process them. For example, an Encrypter can either first encrypt input data with its current key, or first reconfigure its key to a newly supplied one.

As security is typically assessed in reference to some attacker, our language also provides an Attacker process. This process has knowledge, i.e. a set of data elements, which it can extend by guessing new elements (capturing predictable values, but also mechanisms outside of the model such as social engineering), deriving elements from already known ones, or learning data elements it receives as input. By default, an attacker is omnipotent: it can guess any data element as well as derive any element from any other data element. It is up to the designer to explicitly constrain this in a reasonable way.

\section{Networks}

To model more complex behaviour multiple process instances are combined into a single network. In such a network, channels connect an output queue of one process with the input queue of another process, allowing the former to communicate 
TABLE I

SOME PROCESSES HAVE A SPECIFIC GRAPHICAL NOTATION.

\begin{tabular}{lll}
\hline Process & Symbol & Description \\
\hline Collector & $\begin{array}{l}\text { Collects the first data element from each of its input } \\
\text { queues. }\end{array}$ \\
Copier & Copies its input to each of its output queues. \\
Jork & $\begin{array}{l}\text { Outputs input to a non-deterministically selected } \\
\text { output queue. }\end{array}$ \\
\hline
\end{tabular}

its outputs to the latter. A channel propagates its input instantaneously without any failure or data loss; modelling less-thanideal situations requires introducing additional processes.

The state of a network is the combined state of all contained processes and evolves over time in discrete, non-deterministic update steps consisting of two sub-phases: (1) every process may perform an internal transition or do nothing; (2) every channel may propagate its available input data or do nothing. Due to the non-determinism, a given network state can evolve into many different states a single step.

\section{Assumptions}

As described so far, processes and networks behave fairly non-deterministic. While this allows to model a wide range of behaviour, this non-determinism most likely needs to be constrained to match the design at hand. This can be achieved by adding so-called assumptions on elements in a network.

For example, one could constrain an Attacker process to only derive from encrypted data if it also knows the correct cryptographic key. Such an assumption could be warranted if strong encryption is used. Assumptions need to be explicitly stated to trigger a validity check for the design at hand.

\section{E. Notation}

Notation-wise, most processes are depicted as rounded rectangles labelled with their type, with the exception of oftenused auxiliary processes that have designated symbols (cf. Table I). Data that is part of a process's state is depicted between curly braces. Finally, queues are not explicitly shown, but can be explicitly named if they have a specific purpose, e.g. the key queue of an Encrypter is indicated by $\mathbf{K}$. Channels are visualised using arrows between processes (or their queues, if explicit) with the arrow's direction indicating the flow of data.

\section{COMPREHENSION USER STUdy}

We have performed a study focussed on the comprehension of our language's building blocks, individually as well as their combinations, by security novices. In other words, this study assesses whether the foundations of our modelling language are comprehensible, a prerequisite before one can accurately evaluate the comprehensibility of larger designs (such as the one presented in Section V). Furthermore, we also cursory investigated whether security novices are able to actively apply our modelling language themselves. More specifically, we focus on the following research questions:

- RQ1.1 Do participants comprehend the individual building blocks provided by the modelling language?

- RQ1.2 Do participants comprehend models where multiple building blocks have been tied together?

- RQ1.3 Do participants comprehend models where security mechanisms and an attacker are intertwined?

- RQ2.1 Can participants use the language to express an informally described situation?

All study material (e.g. tutorial, tasks, solutions, raw data, analysis scripts) is available on the companion website [19].

\section{A. Study Design}

We performed our comprehensibility study as part of a master level university course on software architecture. The study consisted of two main parts, separated by a few days: a tutorial lecture about the language, and a lab session to fill out a questionnaire. Furthermore, the participants were asked to fill out an entry survey (between the lecture and lab session) and an exit survey (after the lab session) to obtain extra information.

a) Participants: A large majority of the 105 participants (92\%) follow the course as part of a two year computer science master program, $15 \%$ of which follow a secure software specialisation. Based on the entry survey, $55 \%$ of the participants rate their own security expertise as novice, $43 \%$ as intermediate, and only $2 \%$ as security experts. Furthermore, a slight majority of the participants (52\%) has software development experience outside their university courses, mostly from summer jobs, internships and personal projects. We believe this forms a suitable population for the purpose of our study.

Incentives The tasks performed as part of the study were a mandatory part of the course, and participants were graded on commitment (attending the tutorial lecture, the lab session, and the online surveys), as well as performance.

Informed consent The participants were aware they participated in a study from the beginning, without disclosing the precise research questions. Participants had the opportunity to opt out of the study at any point; this affected neither their grades nor the amount of work to perform. No-one opted out.

b) Tutorial: The participants were given a two hour tutorial, containing a brief introduction to software security and a detailed explanation of our language. In this tutorial, all data types and processes were explained, as well as the network semantics and the use of assumptions. The tutorial did not touch upon or even mention the underlying formalisation.

c) Lab session: The participants' principal task was to individually complete a questionnaire concerning our language during a supervised lab session for a maximum of 2.5 hours. The entire lab session was carried out on paper, to prevent participants from communicating electronically. Every participant received a cheat sheet on the language, as well as a copy of the slides used during the tutorial lecture. 
The questionnaire contains a total of 41 questions, where strongly related questions are grouped under a single question number, e.g. 12a and 12b. The questions can be divided into four categories, matching the research questions, testing the comprehensibility of (a) individual building blocks (20 questions), (b) small networks (6 questions), and (c) security-specific aspects of small networks (10 questions), and (d) testing the ability to construct models (5 questions). The comprehensibility questions are multiple choice questions, where one or more answers are correct (e.g. Figure 1). Furthermore, a 'Don't know' option is always available to distinguish questions where a participant doesn't know the answer from unanswered ones. For some questions (especially yes/no questions), participants also have to write down the reasoning that they have used to select their answer.

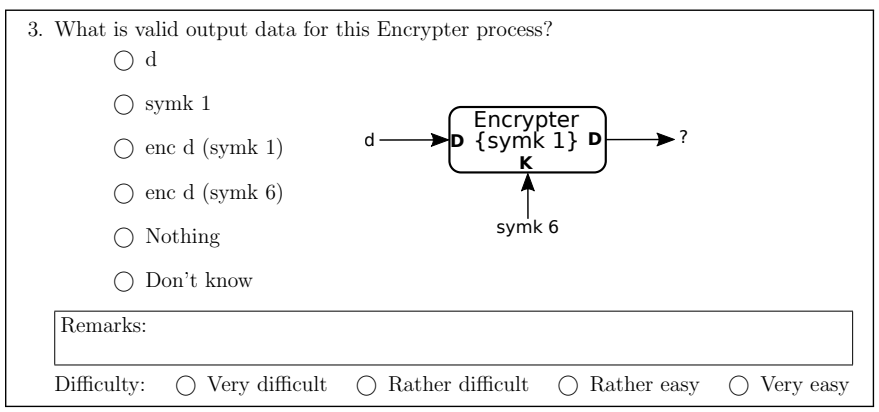

Fig. 1. Example question on the comprehensibility of the Encrypter process

A small number of open questions require the participants to create a network, and state any necessary assumptions, based on an informal description (5 questions). These questions allow a rudimentary assessment of how well the participants can actively apply our language, although this is not the main focus of our study.

Finally, the participants are asked to rate the perceived difficulty of each question on a 4-point scale ('Very difficult', 'Rather difficult', 'Rather easy', 'Very easy'), and they can optionally provide remarks on their answer.

d) Data collection: The participants' answers, along with any remarks, were manually entered into a spreadsheet which was subsequently exported to CSV files. Questions containing free text, such as those requiring the participants to provide their reasoning, as well as open questions, were manually coded using codes capturing, for example, whether a common error occurred in the solution. This coding contains all relevant information of such answers, therefore we did not explicitly recreate any drawn models. The set of codes was not fully determined upfront, but was allowed to grow throughout the analysis, e.g. when an unanticipated common error emerged.

The entry and exit surveys were executed using Google Forms and also exported to CSV files.

To protect the participants' privacy, all identifying information (e.g. name and student number) was immediately replaced by anonymous research ID's.

e) Measured data: In order to quantify how well the participants comprehend the language, we use a scoring func- tion that deals with partially correct multiple choice answers. This function fulfils the following requirements: (1) only fully correct answers get a maximum score; (2) more correct answers (with the same number of incorrect ones) lead to a higher score; and (3) more incorrect answers (with the same number of correct ones) lead to lower score. To avoid negative scores, we cap the minimum score at 0 , yielding the formula score $=\max \left(0,\left(S^{+}-S^{-}\right) / N^{+}\right) \times 100 \%$, where $S^{+}$counts the correctly selected answer options, $S^{-}$the wrongly selected ones, and $\mathrm{N}^{+}$the total number of correct options. The answers to open questions are manually analysed and rated either 'Fully correct', 'Partially correct' or 'wrong'.

\section{B. Results}

In this section, we report on the main insights that arose from analysing the collected and measured data.

1) RQ1.1: Comprehending individual building blocks: The 20 questions concerning RQ1.1 focus on the comprehension of our language's individual building blocks. Across all questions, the participants score rather well, with a mean score of $87 \%$ and a minimum score of $41 \%$. This first, crude, result seems to indicate that at least isolated building blocks are fairly easy to correctly comprehend. A closer look into the individual questions reveals that the encountered problems are confined to a small number of questions, thus building blocks.

The language's strong non-determinism, while expressive, seems to confuse about half of the participants. More specifically, when a process can 'choose' between multiple operations at some point, these participants seem to prefer processes to perform (re)configuration before its main functionality. For instance, they expect that an Encrypter first re-configures its cryptographic key before encrypting any new data when both a new key and data is available. Note that the opposite (a preference for functionality before re-configuration) is rare.

Intuition and formal semantics also seem to clash on the omnipotent knowledge gathering of the attacker. While most participants (64\%) do grasp that an attacker by default can guess or derive any data element, the remaining participants made errors when asked what data given, unconstrained, attacker can get to know. A subset of them seemed to implicitly constrain the abilities of the attacker, which can be detrimental for the security of the software under design, as possible attacks can go unnoticed. An attacker that is omnipotent by default forces designers to explicitly constrain it, and thus reason about the intended attacker model.

A less prevelant issue concerns a mismatch between the definition of data equality and the interpretation thereof by the participants. For example, by our definition, private key prik 1 and public key pubk 1 are distinct data elements, but a minority of the participants (10\%) considered the possibility of them being equal. This may imply that they expect a common representation underlying all data types (e.g. a byte array) that allows equality across different types.

Finally, the Business process with intricate rules on which data it can output, is one of the more complex processes in the modelling language. This complexity is reflected in the fact 
that less than half of the participants (46\%) gave a correct answer on the corresponding question. Such more complex building blocks thus negatively impact comprehension, it seems advisable to avoid them unless absolutely required.

2) RQ1.2: Comprehending combined building blocks: Our second research question concerns the comprehension of processes tied together in a single network without explicitly considering security implications (6 questions). In comparison to RQ1.1, the overall mean score is lower $(79 \%)$, indicating that the combination of individual concepts poses extra challenges.

A deeper investigation reveals that one question (with a mean score of $30 \%$ ) is the main culprit. This question presents a simple network in which data elements can loop indefinitely when transmitted between two processes. Consequently, transmitted data elements can arrive in a different order and some elements may not even arrive at all as they loop indefinitely. Although most participants $(63 \%)$ do see that the order of the sent data elements can change due to the loop, only a minority $(30 \%)$ also catches the possibility for data elements to loop indefinitely. Investigating the provided remarks reveals some participants interpret a network's non-determinism in a more probabilistic way. More specifically, they expect that the probability of data elements staying in the loop reduces over time, i.e. it reduces with each network step, going to zero in the limit. The formal semantics contains no probabilistic component whatsoever, though.

3) RQ1.3: Comprehending security aspects: This third research question focuses on comprehending the security aspects of a model (10 questions). The results for this research question are similar to those for the previous one, with a comparable mean score $(77 \%)$.

Our earlier observation that the omnipotent attacker is challenging to fully grasp is confirmed. For one question, whose correct answer relied on the fact that an attacker (when unconstrained) can immediately guess all (including encrypted) data, the reasoning of a slight majority of participants $(57 \%)$ reveals that they use multiple steps for the attacker to obtain an encrypted data element, namely first guess the plaintext data, and then derive the encrypted version of that. While intuition-based reasoning about omnipotence is often valid and sufficient, it can cause possible attacks to be missed. For example, a (reasonable) assumption about the confidentiality of encryption keys and strength of the encryption algorithm would thwart the multi-step attack, even though the attacker might still be able to obtain the encrypted data in another way.

Furthermore, an important observation is that wording is very important, both when formally defined concepts are used in an imprecise context, as well as when formulating test questions. For example, a particular question about an attacker learning 'by observation' was answered correctly by only $4 \%$ of the participants. Even with clarifications in the question about what 'by observation' means, most participants (96\%) used a different interpretation than the intended one.

4) RQ1 Summary: With a mean score of $83 \%$ (Table II) across all 36 questions for RQ1, the participants appear to comprehend our language's individual building blocks and
TABLE II

SUMMARY SCORES FOR RQ1.1, RQ1.2, RQ1.3, AND RQ1 AS A WHOLE.

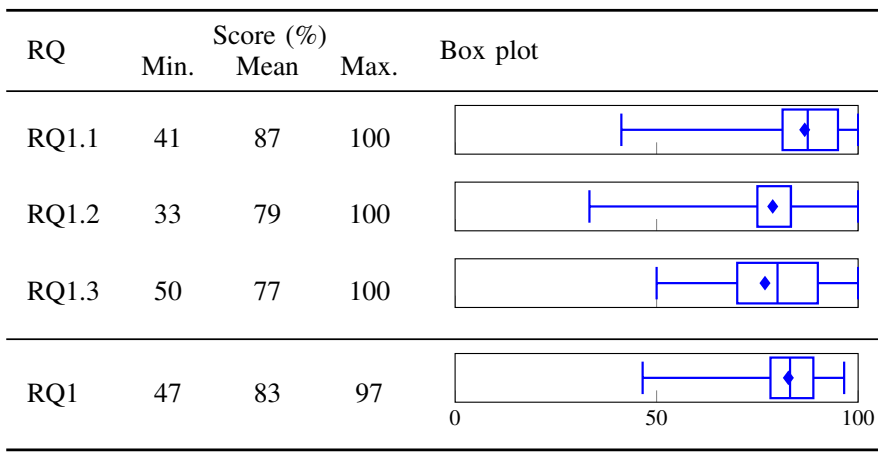

their collaboration in small models rather well. Despite these encouraging results, some recurring issues surfaced. In order to attain the language's full potential in terms of comprehensibility, these issues should be dealt with.

For non-determinism, the fifty-fifty split in interpretations makes it hard to formulate an advice based on the data. Nevertheless, we believe that limiting non-determinism, thus expressiveness, is undesired just to increase comprehension; that about half of the participants do grasp the non-deterministic process behaviour indicates that designers can deal with it, although extra emphasis during training seems advisable.

The attacker's omnipotence severely impedes the comprehension of models, yet from a security perspective this behaviour is crucial to not overlook relevant attacks and assumptions. Several participants did remark that the omnipotence forces one to reason about the attacker's abilities, but doing this in an exhaustive manner is challenging. A possible, partial solution could be to pre-define a set of common attacker types with known, explicit assumptions.

Finally, the participants' misinterpretation of data equality reveals a deeper problem in our language. For instance, it is possible that due to, e.g., misconfiguration public and private keys are the same, but this situation is excluded by our definition of data equality. Such implicit assumptions should be avoided as they mask possible security issues, thus attacks, and thereby jeopardise the results of a security assessment.

5) RQ2: Constructing models: In order to rudimentary gauge to what extent the participants can actively apply our language, we also included some open question requiring the participants to translate an informal description into a network and, if deemed necessary, assumptions. Overall, most resulting designs $(52 \%)$ have been judged partially correct, whereas fully correct design are rather rare (except for one question). As expected, actively using our language thus appears to be quite a bit more difficult than comprehending a given model.

An analysis of the participants' models reveals three recurring problems. First, participants tend to overlook some language idiosyncrasies. For instance, the Store process replies with a collection to read requests, which must be decomposed before it is useful, which many participants neglect to do. Tool support can help to avoid such errors. 
Second, while most participants tend to specify the obvious required assumptions, only few provide an exhaustive set of assumptions. While incomplete assumptions would be discovered when formally proving properties, this is beyond the expertise of most designers.

Finally, participants tend to define new, unexisting processes for modelling more complex logic instead of using those already offered by the language. For example, one of the questions required them to model a DNS spoofing attack. Over one-third of the participants $(35 \%)$ used a non-existing DNS process for the server. Support for hierarchical process composition, allowing to build new ones using already existing processes, might cater this need.

6) Further observations: Both the entry and exit survey asked the participants to rate their perceived difficulty of understanding the language. After the tutorial (but before the lab), $92 \%$ considered it 'rather easy' or 'very easy' to understand, and nobody answered 'very difficult'. After the lab session, results were similar: $93 \%$ of the participants now agree or strongly agree that it was easy to understand. Interestingly, we have not observed a correlation between the participants' perceived understanding and their final score.

A small portion of the participants $(10 \%)$ did not attend the tutorial lecture. These participants still achieved a good overall mean score of $81 \%$ (with $67 \%$ and $92 \%$ as the extremes), and not attending the lecture had no statistically significant impact on the final score. Because these participants could have studied the slides before the lab, we cannot conclude that the tutorial was superfluous, and that our language is comprehensible without training. Furthermore, when asked in the exit survey whether the cheat sheet alone would have been enough to answer the questionnaire, $79 \%$ of participants answered negatively.

In summary, we can conclude that, given a relatively limited tutorial, the participants comprehended the building blocks of our language pretty well. Based on feedback from the participants, as well as our intuition, we hypothesize that three key factors mainly contributed to this result: (1) the simplicity of the building blocks of our language; (2) a visual rather than textual representation (far removed from the notation employed by many formal methods), lending itself well to future tool support; and (3) the fact that the formal underpinnings were completely hidden from the participants, with a focus on intuitive explanations. We strongly believe that more attention to the comprehensibility of languages such as ours is pivotal in order to get them accepted by the broader software design community.

\section{Threats to Validity}

The participants learned the language solely through the lecture and material we provided. Therefore, one can wonder whether we are just evaluating the quality of the given lecture and provided material. When assessing comprehensibility, this factor definitely has an influence. The fact that the participants were able to obtain good scores demonstrates at least that it is possible to learn our language relatively fast. The main focus of the lecture was on explaining individual language elements, which might have contributed to the higher scores on this topic (cf. Section IV-B4). Furthermore, some participants stated in the entry survey that they would have liked more examples of networks.

As indicated by some participants, solving more tasks may improve understanding. Such learning effect is to be expected, and we anticipated this by roughly ordering the questions according to expected difficulty. The difficulty ratings for each question provided by the participants confirm this; they start at very easy and evolve towards difficult near the end. Very difficult ratings were rare. Furthermore, participants had the time and opportunity to correct previous answers when gaining new insights along the way.

Another potential threat is that the study was entirely performed on paper, and was bounded in time. This resulted in some remarks about the time participants spent riffling through papers. While this is indeed artificial, it was our deliberate choice in order to avoid communication and ensure accurate data. Moreover, on average the participants completed the questionnaire in just under 2 hours, with only few participants fully using the allocated 2.5 hours of the lab session.

Finally, the participants in our study are master students rather than professionals. Based on $52 \%$ of them having real-world development experience, only $2 \%$ identifying as a security expert, and previous empirical research on using students as participants [20], [21], we do believe that they are representative for part of the broader population to which security views must be communicated. More specifically, we believe our participants are representative substitutes for junior, security novice software developers. Comprehension from the perspective of non-technical stakeholders, e.g. managers, requires further research.

\section{A Realistic Authentication Model}

To assess the language's practicality from a security specialist's perspective, we have used it to create a precise, realistic model of password-based authentication, including sessions, user registration, two-factor authentication, password changes, and password resets, extending our simple example in [4] based on best practices from OWASP [22], [23], [24], [25].

A model such as the one created here can serve multiple purposes. It can be used to document the security aspects of a specific software system for future reference, for communication with stakeholders, or for performing a precise analysis of its properties. Similarly, a model of commonly used solutions can be used to precisely express reusable knowledge.

\section{A. Model excerpts}

Because the resulting model itself is too large to present in this section, we limit ourselves to highlighting some excerpts from it; the whole design is available on the companion website [19]. Graphically, we use partial rectangles $(\sqcup)$ to abstract parts of the design that are not included. 
Secure account storage: Besides a username and password, the account information stored upon registration is extended with a phone number for two-factor authentication, as well as an email address and two security questions along with their answers for a proper password reset feature (Figure 2).

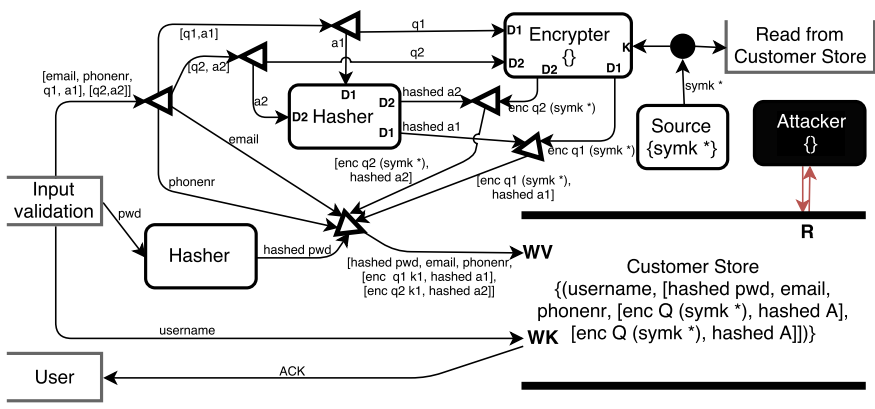

Fig. 2. Excerpt for secure account storage

As the Attacker in this model is able to read from the Customer Store, e.g. as a consequence of a SQL injection vulnerability, we must protect the contained information. Therefore, instead of storing the passwords as plaintext, we store their hash values [23]. The security questions are stored in encrypted form [24, Step 4]. For the answers to the security questions, only their hash values are stored [24, Step 4]. Email addresses and phone numbers are stored in plaintext, but these could also be encrypted if necessary. The explicit Hasher and Encrypter processes in the model (Figure 2) capture the presence of the mentioned security precautions.

Two-factor authentication: A common extension to the standard username/password authentication is adding extra factors [22]. A good second factor is transmitted or created via an out-of-band channel, for example a one-time code sent via text message, or generated using a specialised smartphone app. Here we show how the text message case can be modelled (Figure 3); the smartphone case is also part of the full model.

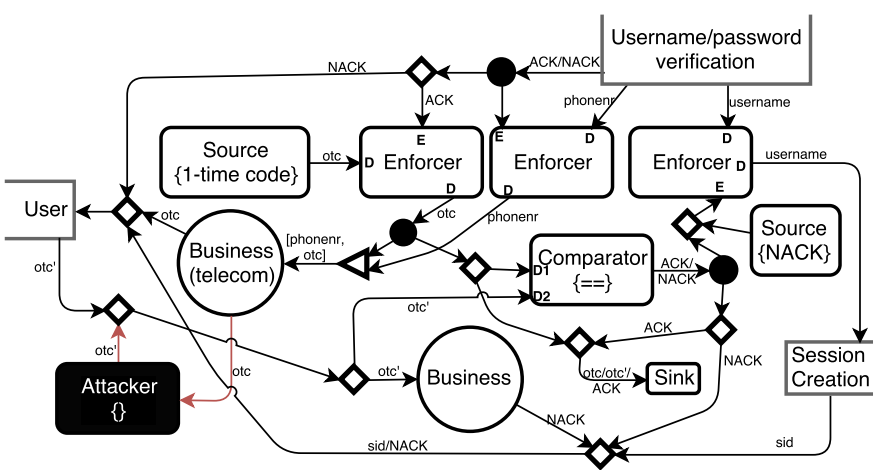

Fig. 3. Excerpt for two-factor authentication

One-time codes used as second factor should be impossible to guess, which can be specified using assumptions on the Attacker and the Source process that generates these codes. Furthermore, each code is valid for only a limited amount of time, usually no more than a few minutes. This is essentially modelled using two synchronised Fork processes, where one only forwards its input when a code arrives at the other in a timely fashion, and one Joiner process to discard the original request when the sent code times out.

The three Enforcers in the model (Figure 3) are used to ensure that just a single code is sent when the first factor has been successfully verified, and that sessions are created only when the second factor has been successfully verified.

The extra authentication factor also influences the attacker model. Clearly, it reduces the possibility of a successful bruteforce attack, but due to the insecure nature of regular text messages, an attacker with sufficient resources could obtain transmitted one-time codes by intercepting the telephone network traffic. This is modelled by explicitly connecting the telecom provider (Business process) to the Attacker (Figure 3); the consequence of doing this given the semantics of the language will simply be that the second factor provides no additional protection. Whether such an attack is reasonable, and thus worth protecting against, depends on the system at hand, and likely requires some sort of risk analysis.

Security questions: Security questions allow to increase the security of the password reset functionality [25, Step 1]. Verifying whether a User provides the correct answers to his or her security questions is rather straightforward, by calculating the hash values of the User's answers and comparing them against the hashes stored by the system.

The omnipotent attacker model immediately allows the Attacker to guess all questions and corresponding answers, thereby breaking the protection. Adding a restriction that an attacker cannot even guess one valid question/answer pair might be unrealistic, however (what if the attacker itself creates an account?). Therefore, as a compromise, it is assumed that there exists a finite set of pairs that can be guessed by the Attacker, e.g. a list of 'easy to guess' questions and answers, and that users will not pick combinations from that set.

Furthermore, in order to reduce the possibility of an attacker correctly answering security questions by brute-force, we limit the number of times a user (or attacker) is allowed to try [25, Step 2]. A Latch process (which is a simple memory cell) serves as the reification of the attempt counter.

\section{B. Experience}

We have succeeded in modelling a realistic two-factor authentication mechanism that also encompasses sessions, user registration, password changes, and password resets in our modelling language. While this is an encouraging result, we do not consider our language ready for prime time just yet.

We found using our pre-defined building blocks, e.g. processes and data types (with their formalised semantics) advantageous over the constructs provided by general purpose languages such as UML or DFD. Modelling with the predefined processes at times also triggered us to explicitly reason about possibilities we had not anticipated (e.g. the possibility to use the change password functionality to re-create a deleted user account from an active session). 
Similar to the experience of our study participants, the semantics of processes was at times cumbersome to work with, though, for example parsing the replies of a Store. In concordance with the participants, we feel there is a need to encapsulate recurring behaviour into higher-level processes.

Furthermore, it turned out to be challenging to define a comprehensive set of assumptions to attain the desired security properties, e.g. constraining the attacker or limiting the non-determinism of Disperser, Fork and Collector processes. Moreover, assumptions are not well integrated into the language. This is partially mitigated by working directly with the formal underpinnings, yet this carries a significant cost concerning the required expertise. Extracting and re-using common behaviour and assumption patterns is therefore an approach worth investigating.

Finally, we also faced some difficulties with modelling time-related aspects. For example, the above-mentioned use of Forks and assumptions to model invalidating the second factor code after a timeout, or the password reset feature which requires invalidating any reset token sent to the user after a certain amount of time. Better support for dealing with time at a more abstract level could be advantageous.

\section{CONCLUSION}

In order to tackle security from the early software development phases, as advocated by the security by design principle, a lingua franca for security modelling should be available to designers. Such a language must reconcile the high level of precision required by security experts with the ability to communicate the resulting models to a broader audience.

We have investigated whether our security modelling language achieves this delicate balance. Therefore, we have performed a descriptive study in which 105 participants were asked to solve a number of controlled tasks focusing on comprehensibility. Furthermore, we have modelled a realistic version of the well-known username/password authentication mechanism, comprising sessions, two-factor authentication, user registration, users changing their password, and account recovery in case of a forgotten password using our language.

The results demonstrate (1) that novice software designers, after limited training, can attain a good comprehension of our language's individual building blocks, as well as models combining a limited number of building blocks; and (2) that our language can be used to model realistic security solutions.

Albeit these are positive results, which seem to make our language a promising candidate for the position of lingua franca, it is not yet ready for prime time, and follow-up research is necessary. For instance, our own experience, as well as that of several study participants indicate that their is an unfulfilled need for composition and abstraction, facilitating easier construction of sound models while also keeping their size more manageable. Furthermore, an improved graphical notation, along with tool support, can shield the designer from several idiosyncrasies currently present in our language. Also, now that it has been established that the foundations of our language are comprehensible to security novices, the comprehensibility of larger, realistic models such as the one we have created warrants further investigation.

\section{REFERENCES}

[1] Microsoft, "Microsoft security bulletin ms15-011." [Online]. Available: https://technet.microsoft.com/en-us/library/security/ms15-011.aspx

[2] J. Jürjens, Secure Systems Development with UML. Springer, 2004.

[3] N. Moebius, W. Reif, and K. Stenzel, "Modeling security-critical applications with UML in the SecureMDD approach," International Journal On Advances in Software, vol. 1, no. 1, pp. 59-79, 2009.

[4] A. van den Berghe, K. Yskout, R. Scandariato, and W. Joosen, "A Model for Provably Secure Software Design," in IEEE/ACM 5th International FME Workshop on Formal Methods in Software Engineering, 2017.

[5] P. T. Devanbu and S. Stubblebine, "Software engineering for security: A roadmap," in Proceedings of the Conference on The Future of Software Engineering, ser. ICSE '00, 2000, pp. 227-239.

[6] P. H. Nguyen, M. Kramer, J. Klein, and Y. Le Traon, "An extensive systematic review on the model-driven development of secure systems," Information and Software Technology, vol. 68, pp. 62-81, 2015.

[7] A. van den Berghe, R. Scandariato, K. Yskout, and W. Joosen, "Design notations for secure software: a systematic literature review," Software \& Systems Modeling, vol. 16, no. 3, pp. 809-831, Jul 2017.

[8] T. Heyman, R. Scandariato, and W. Joosen, "Reusable formal models for secure software architectures," in Software Architecture (WICSA) and European Conference on Software Architecture (ECSA), Joint Working IEEE/IFIP Conference On, 2012.

[9] M. Almorsy and J. Grundy, "SecDSVL: A Domain-Specific Visual Language to Support Enterprise Security Modelling," in 2014 23rd Australian Software Engineering Conference, 2014, pp. 152-161.

[10] M. Giordano, G. Polese, G. Scanniello, and G. Tortora, "A system for visual role-based policy modelling," Journal of Visual Languages \& Computing, vol. 21, no. 1, pp. 41-64, 2010.

[11] T. DeMarco, Structured Analysis and System Specification, 1978.

[12] A. Shostack, Threat Modeling - Designing for Security. John Wiley \& Sons, 2014.

[13] A. W. Roscoe, "CSP and determinism in security modelling," in IEEE Symposium on Security and Privacy, 1995, pp. 114-127.

[14] G. Lowe, "A hierarchy of authentication specifications," in Proceedings 10th Computer Security Foundations Workshop, 1997, pp. 31-43.

[15] M. Abadi, B. Blanchet, and C. Fournet, "The Applied Pi Calculus: Mobile Values, New Names, and Secure Communication," Journal of the ACM (JACM), vol. 65, no. 1, pp. 1:1-1:41, 2017.

[16] B. Blanchet, "CryptoVerif: A Computationally-Sound Security Protocol Verifier," Tech. Rep., 2017.

[17] —, "Automatic Verification of Security Protocols in the Symbolic Model: The Verifier ProVerif," in Foundations of Security Analysis and Design VII: FOSAD, 2014, pp. 54-87.

[18] P. Bettassa Copet, A. Pironti, D. Pozza, R. Sisto, and P. Vivoli, "Visual model-driven design, verification and implementation of security protocols," in Proceedings of IEEE International Symposium on High Assurance Systems Engineering, 2012, pp. 62-65.

[19] Companion website. [Online]. Available: https://people.cs.kuleuven.be/ alexander.vandenberghe/secdev18.html

[20] M. Höst, B. Regnell, and C. Wohlin, "Using students as subjects - a comparative study of students and professionals in lead-time impact assessment," Empirical Software Engineering, vol. 5, no. 3, pp. 201214, 2000.

[21] I. Salman, A. T. Misirli, and N. Juristo, "Are students representatives of professionals in software engineering experiments?" in Proceedings of the 37th International Conference on Software Engineering-Volume 1, 2015, pp. 666-676.

[22] E. Keary, J. Manico, T. Goosen, P. Krawczyk, S. Neuhaus, and A. Morales, "Authentication Cheat Sheet." [Online]. Available: https://www.owasp.org/index.php/Authentication_Cheat_Sheet

[23] J. Steven and J. Manico, "Password Storage Cheat Sheet." [Online]. Available: https://www.owasp.org/index.php/Password_Storage_Cheat_ Sheet

[24] K. Wall, "Choosing and Using Security Questions Cheat Sheet." [Online]. Available: https://www.owasp.org/index.php/Choosing_and_ Using_Security_Questions_Cheat_Sheet

[25] D. Ferguson, J. Manico, K. Wall, J. McGovern, and W. Philip, "Forgot Password Cheat Sheet." [Online]. Available: https://www.owasp.org/ index.php/Forgot_Password_Cheat_Sheet 